Musées, Patrimoine et Culture scientifiques et techniques

\title{
Par les fruits et légumes, l'interprétation de différents patrimoines
}

\section{Pauline Grison}

\section{OpenEdition \\ Journals}

Édition électronique

URL : http://journals.openedition.org/ocim/357

DOI : 10.4000/ocim.357

ISSN : 2108-646X

Éditeur

OCIM

Édition imprimée

Date de publication : 1 septembre 2008

Pagination : 31-35

ISSN : 0994-1908

Référence électronique

Pauline Grison, « Par les fruits et légumes, l'interprétation de différents patrimoines », La Lettre de I'OCIM [En ligne], 119 | 2008, mis en ligne le 21 janvier 2011, consulté le 02 mai 2019. URL : http:// journals.openedition.org/ocim/357; DOI : 10.4000/ocim.357 


\title{
Par les fruits et légumes, I'interprétation de différents patrimoines
}

\author{
Pauline Grison *
}

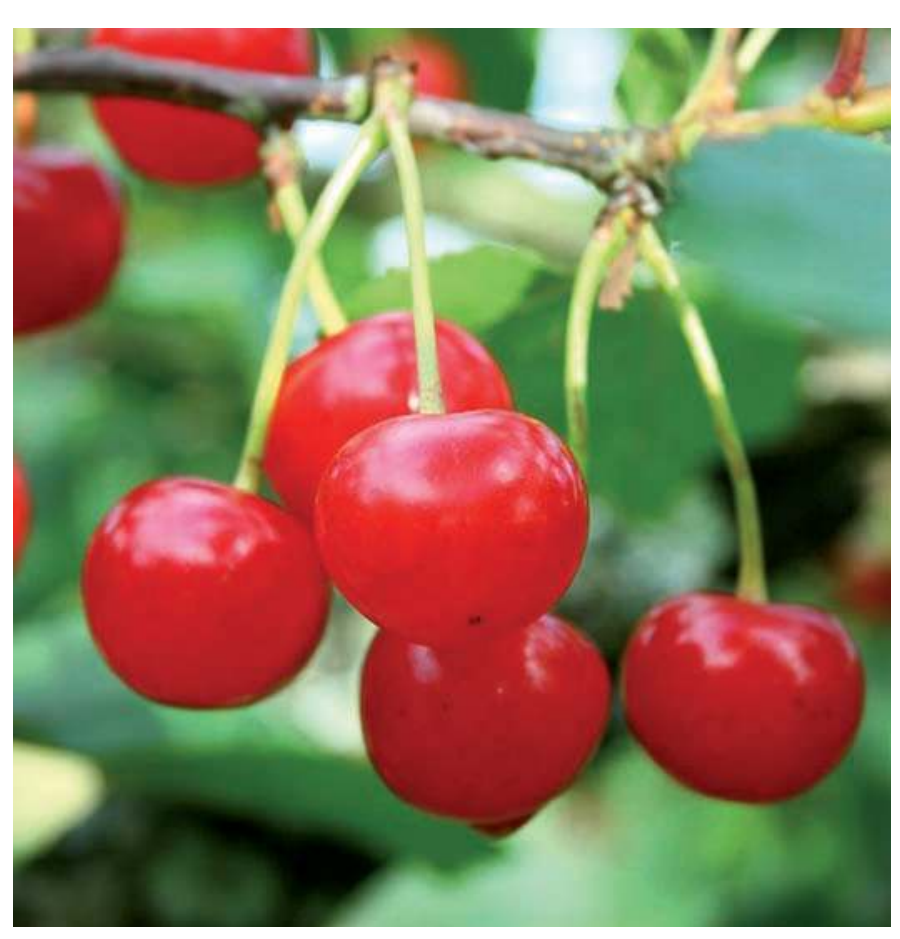

Les fruits et légumes sont au cœur du projet d'exposition, mais c'est leur contextualisation dans un environnement naturel et des activités humaines qui constitue l'objet de l'interprétation.

(C) PEIFL

* Pauline Grison est chargée de mission au Pôle Européen d'Innovation Fruits et Légumes et doctorante au sein du laboratoire Culture et Communication de l'université d'Avignon Pauline.GRISON@peifl.org
À partir de l'analyse d'un programme muséographique organisé autour $d^{\prime} u n$ patrimoine naturel, culturel, technique et industriel lié aux fruits et légumes et qui a choisi l'activité d'interprétation comme forme de médiation, l'auteur montre comment cette démarche spécifique permet de concilier l'exigence de fiabilité attachée à l'institution muséale et le respect des intérêts économiques des acteurs de la filière porteurs du projet.

Epicurium est un projet d'espace muséographique sur les fruits et légumes situé à Avignon. Initié par le Pôle Européen d'Innovation Fruits et Légumes (PEIFL) - pôle de compétitivité de la filière -, ce projet a pour objectif de favoriser une découverte culturelle, scientifique et technique des fruits et légumes. L'exposition permanente proposera une approche pluridisciplinaire, avec comme thématiques centrales l'alimentation, l'agriculture, l'industrie et l'environnement.

Le programme muséographique en cours d'élaboration associe une forme de mise en exposition traditionnelle - dispositifs scriptovisuels et audiovisuels notamment - à des formes de médiation plus proches de celles des centres d'interprétation, qui valorisent une approche sensible et centrée sur le visiteur et l'expérience de visite. C'est sur ce deuxième type de médiation, qui concerne notamment un patrimoine dont la forme ne peut se prêter à une muséification, que nous allons porter notre 
réflexion. Nous souhaitons montrer comment, au stade de la conception d'un projet, s'articulent la réflexion autour du patrimoine à interpréter et des outils à mettre en œuvre pour aider à son interprétation. De quel patrimoine s'agit-il ? Comment le rendre signifiant et perceptible? Telles sont les questions auxquelles nous nous proposons de répondre ici à partir de l'exemple du projet Epicurium.

\section{Un patrimoine vivant}

Bien que la valorisation du patrimoine ne fasse pas partie des objectifs initiaux du projet, c'est la réflexion menée lors de la conception du programme muséographique d'Epicurium qui a mis en évidence la réa lité de tout un patrimoine vivant que l'exposition allait mettre en lumière : un patrimoine naturel, culturel, technique et industriel lié aux fruits et légumes.

Entre nature et culture

Les fruits et légumes sont d'abord une manifestation bien visible d'un patrimoine naturel dont nous sommes à la fois dépendants et garants. La diversité variétale, par exemple, est le fruit d'un processus naturel de diversification des variétés lié notamment au rôle des insectes pollinisateurs, mais aussi à des techniques initiées par l'homme sur imitation de la nature pour répondre à ses besoins. De même, le milieu a conditionné l'adaptation de certaines espèces, qui ont à leur tour conditionné certaines activités humaines ayant elles-mêmes à leur tour des répercussions sur le milieu : c'est le fruit de ces interactions entre un milieu, des plantes et des hommes qui forme les caractéristiques de notre environnement naturel aujourd'hui.

\section{Des goûts et des saveurs}

Dans un tout autre domaine, les fruits et légumes font partie d'un patrimoine culturel méditerranéen. Dans la culture culinaire méridionale en effet, l'attention portée au plaisir alimentaire à travers les saveurs, les arômes, les textures et même les couleurs font des fruits et légumes les symboles d'un art de vivre lié à un certain art du goût et de la simplicité. Alors que la tendance en matière d'éducation nutritionnelle est à une forme de médicalisation de l'alimentation, le défi de l'interprétation consiste à revaloriser la dimension sensorielle et symbolique de ce patrimoine, notamment par un plaisir retrouvé des sens ${ }^{(1)}$.

\section{L'agroalimentaire, entre tradition et modernité} Enfin, les fruits et légumes renvoient naturellement aux activités humaines consacrées à leur production, à savoir l'agriculture et l'industrie. Le territoire du Comtat Venaissin est profondément marqué par le maraîchage et l'arboriculture, avec un paysage quadrillé par des haies de cyprès brise-vent et des canaux d'irrigation issus de la Durance. Un œil

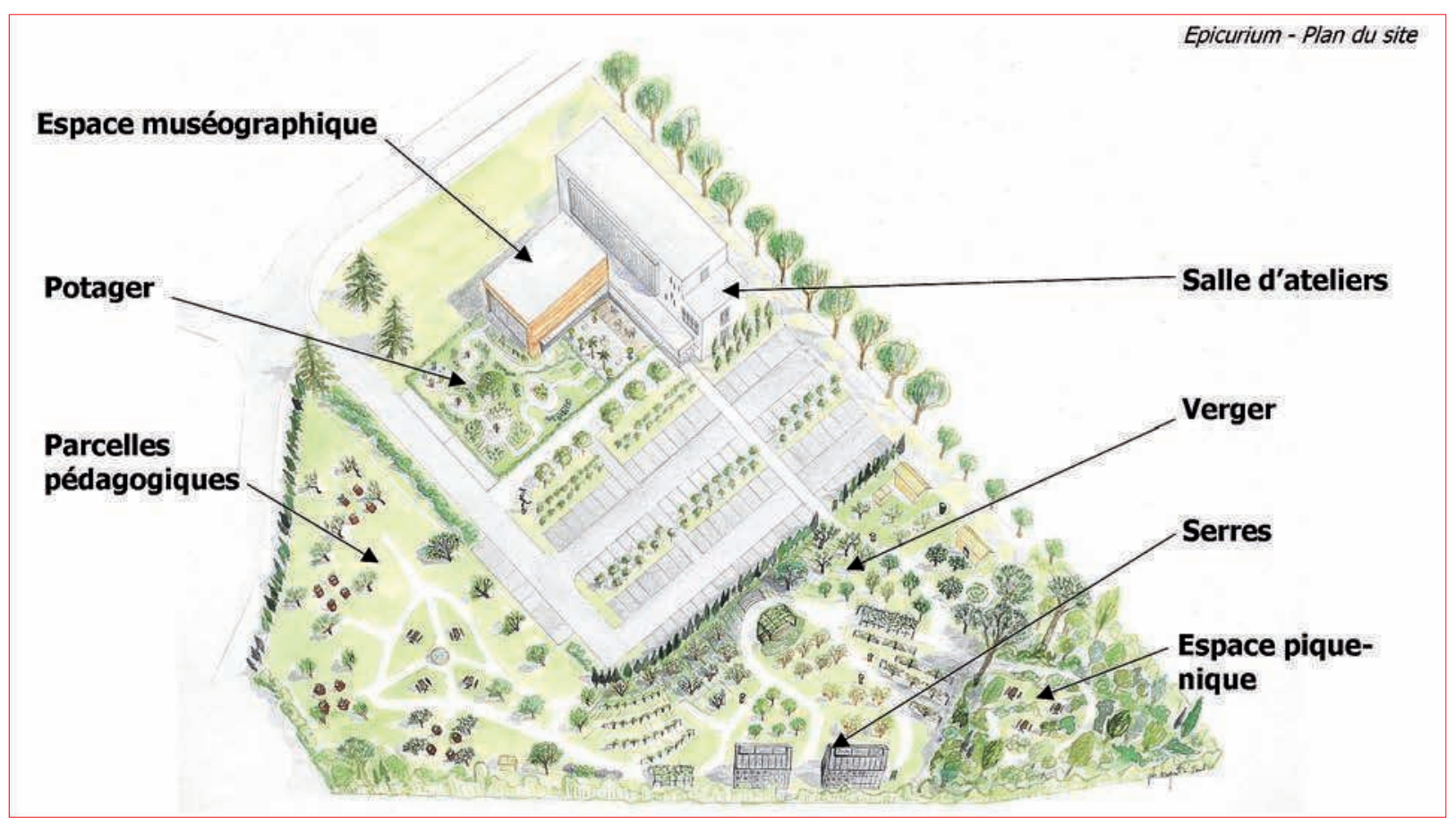

Le futur Epicurium comportera un espace muséographique, un potager, un verger, deux serres et une salle d'ateliers. 
aiguisé et aguerri pourrait en apprendre beaucoup sur l'agriculture d'aujourd'hui, qui allie savoir-faire traditionnels - greffe, taille des arbres, association de cultures... - et techniques nouvelles - lutte biologique intégrée, culture hors-sol ou encore récolte mécanisée. L'industrie agroalimentaire en revanche n'est pas aussi directement visible dans cet environnement, bien que constituant un patrimoine local de taille en Vaucluse. Les usines et les machines sont des boîtes opaques qui laissent transparaître bien peu d'informations, et l'enjeu de l'activité d'interprétation consiste notamment à « ouvrir » ces boîtes aux visiteurs curieux.

\section{Le projet d'interprétation}

Un des postulats de l'activité d'interprétation comme forme de médiation est que les « objets » ne parlent pas d'eux-mêmes ${ }^{(2)}$. Comment rendre ce patrimoine signifiant pour le visiteur ? C'est le travail d'interprétation effectué en amont qui va véritablement construire le patrimoine et le mettre en lumière, avec la définition de clés de lecture qui sont autant fonction des volontés communicationnelles de l'institution que des représentations du visiteur intégrées au processus de conception.

\section{Un angle d'approche...}

En tant que pôle de compétitivité rassemblant des acteurs de l'ensemble de la filière, la volonté du PEIFL est de produire une interprétation de notre environnement naturel et agroalimentaire qui soit actuelle et dans laquelle l'ensemble des acteurs de la filière puissent se reconnaître. Le projet d'interprétation, formé par l'articulation entre le patrimoine et l'angle d'approche voulu, consiste ici à montrer des interactions entre des hommes, des techniques et un milieu, par le prisme des fruits et légumes. Il s'agit donc de l'interprétation d'un patrimoine contemporain, qui peut être appréhendé par la contextualisation d'un « objet » quotidien - les fruits et légumes - dans un environnement naturel, social, économique, technique et culturel. L'enjeu n'est pas d'inciter à une conservation de ces patrimoines, mais de faire prendre conscience de leur existence et de leur réalité, en favorisant une certaine forme de réflexivité dans notre regard et nos actes quotidiens.

\section{... et des clés de lecture..}

Les clés de lecture, qui forment la structure du discours d'exposition, doivent s'appuyer sur les représentations des visiteurs potentiels pour être pertinentes. Prenons l'exemple de l'agriculture. L'objectif du projet muséographique est de faire connaitre le métier de maraîcher et d'arboriculteur aujourd'hui, les techniques de culture, et de sensibiliser à la saisonnalité des travaux et produits agricoles. Or, dans l'imaginaire collectif, l'agriculture est associée aux valeurs de la terre et à son authenticité, ainsi qu'aux savoir-faire et modes de vie qui sont encore ceux des années précédant la révolution agricole amorcée dès les années $1950^{(3)}$. Elle se voit assignée une fonction symbolique décalée par rapport à la réalité, liée au mouvement de retour au naturel et aux angoisses de la modernité alimentaire qui, dans les années 1980, conduit à survaloriser le terroir par opposition à l'industrialisation alimentaire ${ }^{(4)}$. La prévalence de cette représentation de l'agriculture permet de comprendre pourquoi l'emploi de techniques modernes (chimie, génétique, culture sous serre...) et la soumission à des impératifs économiques de production sont souvent perçus comme une menace pour l'agriculture «traditionnelle », faisant basculer ceux qui les emploient dans la catégorie des entrepreneurs et industriels, avec toutes les connotations péjoratives que cela implique. Dès lors, comment faire comprendre ce qu'est l'agriculture aujourd'hui sans se heurter à des représentations qui la ferait la rejeter en bloc par comparaison avec l'agriculture authentique et nourricière de nos imaginaires ?

Une piste est donnée à ce propos par Joëlle Le Marec qui préconise d'utiliser la dépendance au milieu naturel comme entrée pour aborder la technique et

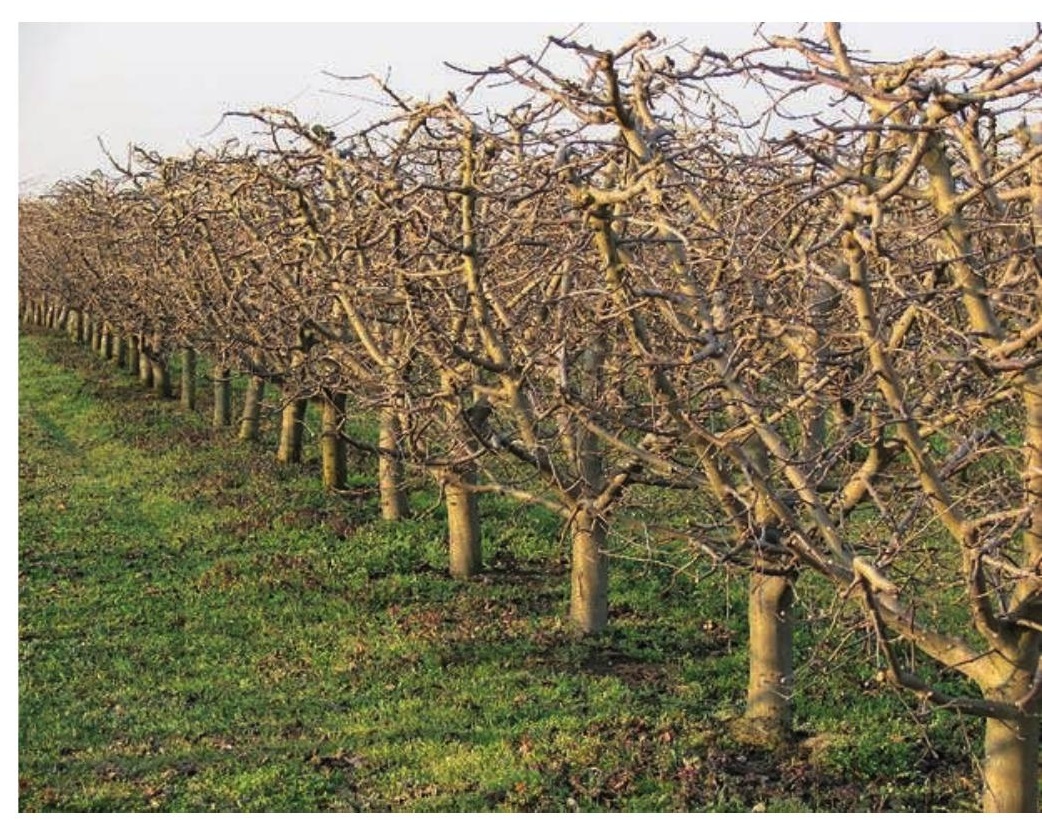

Les espaces extérieurs d'Epicurium - verger, potager et serres seront le lieu privilégié d'une sensibilisation au patrimoine naturel et agricole lié aux fruits et légumes. 
de l'articuler sur des représentations concernant l'homme et son milieu ${ }^{(5)}$. Cette idée est intéressante car la dépendance au milieu naturel - et les tentatives pour s'en affranchir - est un élément clé de l'évolution des pratiques agricoles au cours de l'histoire. Elle constitue, en outre, un moyen d'aborder l'aspect social et économique de la thématique, mais aussi de faire le lien avec l'industrie agroalimentaire qui est aussi, en quelque sorte, un prolongement de l'effort des hommes pour s'affranchir de différentes contraintes naturelles et domestiques. Ainsi, une telle approche peut permettre de donner du sens au patrimoine en respectant tout autant les volontés communicationnelles du concepteur que la nécessité de faire écho à des thématiques susceptibles d'interpeller le visiteur et de l'amener à un questionnement.

\section{... pour un projet de " mise en culture »}

Cette attention portée aux représentations traduit en effet une volonté de "mise en culture » de ce patrimoine, pour reprendre la conception de Jeanmarc Lévy-Leblond d'une mise en culture de la science consistant notamment à mettre en avant, dans l'objet à interpréter, la dimension culturelle susceptible de susciter intérêt et questionnement de la part du visiteur (6). L'articulation entre savoirs scientifiques et savoirs de sens commun, appelés aussi savoirs sociaux, permet d'envisager une culture interprétative commune plutôt que de penser la circulation des représentations entre deux univers sociaux différents ${ }^{(7)}$. Le projet d'interprétation ainsi conçu peut alors entrer en résonance et en dialogue avec les représentations et expériences du visiteur, et atteindre de la sorte son objectif de sensibilisation à un patrimoine.

\section{Les aides à l'interprétation}

De manière plus pragmatique, la dernière étape dans la conception du projet d'interprétation consiste à déterminer les aides à l'interprétation qui vont accompagner le visiteur dans la découverte du patrimoine. Quels dispositifs choisir pour matérialiser les clés de lecture ? Dans le cas d'Epicurium, deux questions principales se sont posées : comment figurer un patrimoine absent sur le site de visite, c'est-à-dire comment mettre en lumière un patrimoine environnant ? Et comment, puisqu'il s'agit d'un patrimoine en grande partie lié au végétal, donc au vivant, permettre d'appréhender de manière sensible ce patrimoine ?

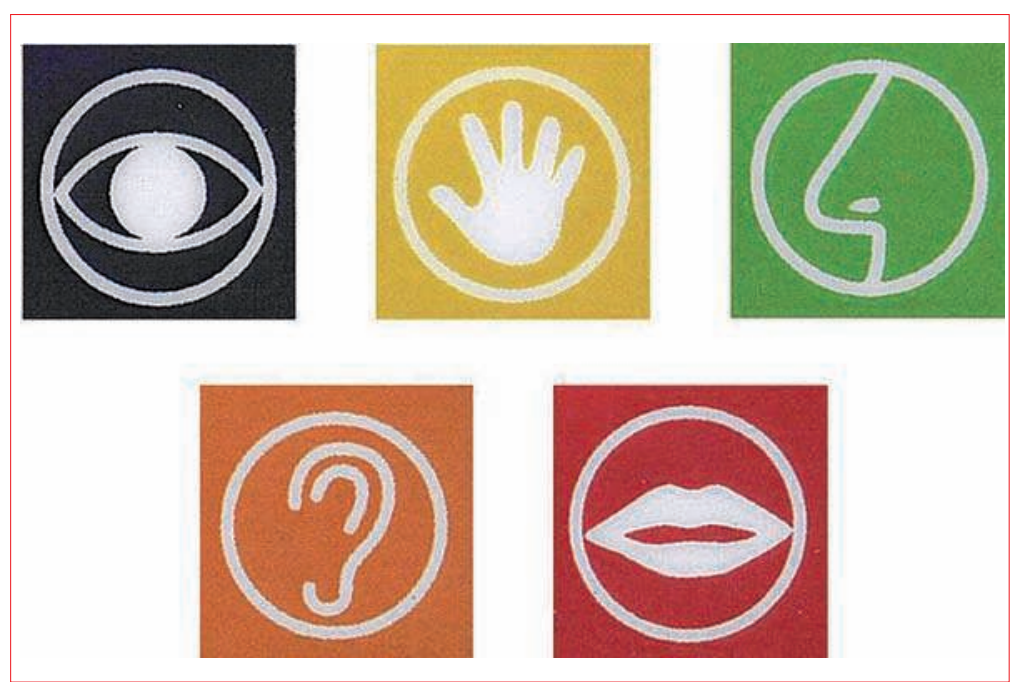

$$
\begin{array}{r}
\text { Ces petits pictogrammes sont des dispositifs } \\
\text { d'accompagnement du visiteur qui favorisent l'appropriation } \\
\text { - et donc l'interprétation - sensorielle de l'exposition. } \\
\text { ○ PEIFL/Nouveau Sud }
\end{array}
$$

\section{La valorisation}

de l'expérience sensorielle de visite

Il nous semble que, dans le cas d'un patrimoine naturel ou d'un patrimoine technique et culturel lié au vivant, rien ne puisse remplacer le contact direct aux choses, l'expérience sensible. Pour un objet comme les fruits et légumes, il a paru important de favoriser une approche multisensorielle de l'exposition, et ce de trois manières différentes. D'abord, la mise en scène des couleurs, des odeurs, des sons, des lumières et du végétal dans l'espace muséographique intérieur permet d'immerger de manière discrète le visiteur dans l'univers vivant des fruits et légumes, avant même qu'il ait parcouru les espaces extérieurs. Ensuite, l'aménagement d'alcôves sensorielles dans l'exposition incite explicitement le visiteur à tester ses cinq sens et à s'interroger ainsi sur le rôle de chacun d'eux dans sa perception du monde. Enfin, le potager et le verger, utilement complémentaires de l'expo sition, sont naturellement le règne des odeurs, des sons et des saveurs. Mais toujours dans l'idée que certains éléments ne parlent pas d'eux-mêmes, une signalétique simple de petits pictogrammes mobiles rappelant les cinq sens permet d'alerter le visiteur et de l'inciter à porter attention à un élément particulier, qu'il s'agisse d'un plant particulièrement odorant ou d'un fruit rugueux par exemple. L'objectif de ce dispositif est d'inciter à une perception plus consciente de notre environnement, avec des suggestions à poursuivre cette démarche au quotidien. 


\section{Le sentier de découverte en plein air}

Les espaces extérieurs d'Epicurium sont conçus comme un sentier découverte, qui remplit tout autant les fonctions de délectation que celles de mise en exposition sensible du savoir : il s'agit en quelque sorte d'une exposition " grandeur nature », par laquelle l'objet de l'interprétation est partiellement recréé pour combler son absence sur le site. Dans le potager par exemple, le visiteur découvre des parcelles consacrées à la diversité variétale. Il traverse ensuite, dans le verger, différents milieux caractéristiques du territoire, avec des oliviers, des pistachiers et des câpriers sur un talus aride et rocailleux, et des vergers irrigués en contrebas. Un peu plus loin, une haie de cyprès doit faire prendre conscience, les jours de Mistral, de l'efficacité de cette fonction d'abri thermique et de brise-vent. Enfin, différentes techniques de conduite des cultures sont montrées, avec par exemple des arbres fruitiers en palissage ou en plein-vent, et une serre qui permet d'illustrer les techniques de culture en sol et hors-sol. Nous pensons que ces éléments d'interprétation, agrémentés d'une signalétique légère, seront un moyen d'apprendre à réobserver le paysage.

\section{La mise en réseau sur le territoire}

Finalement, la visite d'Epicurium peut constituer une sorte de "mise en bouche ", un premier contact avec un environnement que l'on ne soupçonnait pas si proche. La deuxième étape est, pour ceux qui le souhaitent, la visite in situ d'une exploitation agricole ou d'une structure agroalimentaire. Ainsi, l'espace d'interprétation prend tout son sens car il se déploie véritablement sur le territoire, à la rencontre des hommes et du patrimoine vivant.

\section{Interprétation et points de vue}

Les choix de discours et de dispositifs que nous avons présentés ici forment une interprétation parmi d'autres de notre environnement agroalimentaire. Car c'est finalement le propre de toute activité d'interprétation, au sens commun comme au sens muséographique du terme, que d'être l'aboutis sement d'une démarche singulière de médiation émanant de la subjectivité d'une institution. Il reste à ce propos un point que nous n'avons pas mentionné jusqu'ici, à savoir le caractère parfois sensible des thématiques évoquées, comme par exemple l'environnement ou l'alimentation qui sont au cœur de tensions entre préoccupations économiques, sociales, sanitaires et écologiques.

Dans le cas d'Epicurium, qui émane d'un pôle de compétitivité dont la vocation première est d'être au service des acteurs de la filière pour accélérer son développement économique par l'innovation, le traitement muséographique de sujets qui dépassent la dimension patrimoniale pour s'inscrire dans le domaine des relations entre sciences, techniques et société, pose question. Comment concilier la garantie de fiabilité attachée à l'institution muséale et la nécessité de respecter les intérêts économiques des acteurs professionnels et scientifiques du pôle de compétitivité ? Nous pensons que c'est justement le recours à la démarche spécifique des centres d'interprétation qui peut aider à relever ce défi, en offrant des outils pour comprendre, sentir et s'interroger plutôt que des connaissances pour expliquer ou argumenter. Par l'attention portée au visiteur, à ses attentes et à ses représentations, la médiation proposée par les centres d'interprétation reconnaît et favorise la capacité interprétative du visiteur, permettant ainsi la formation d'une pluralité de points de vue dans l'activité de réception. Tout l'enjeu du processus de conception réside alors dans la recherche d'un juste milieu entre la volonté de transmettre un message - par des clés de lecture qui sont fonction des visiteurs potentiels, mais aussi des objectifs communicationnels de l'institution - et celle de favoriser le libre jeu de l'interprétation dans l'activité de réception.

\section{Notes}

(1) Voir notamment Poulain, J.-P. Manger aujourd'hui. Attitudes, normes et pratiques. Toulouse : Éditions Privat, 2002, pp. 191-200.

(2) Voir notamment le dossier consacré à l'interprétation, la Lettre de l'OCIM, n61, 1999.

(3) Le Marec, J. Évaluation préalable (synthèse) : agriculture, cellule Évaluation, Cité des Sciences et de l'Industrie, Paris, 1992 ; Mayaud, J.-P. Gens de l'agriculture, la France rurale 1945-2005. Paris : Éditions du Chêne, 2005

(4) Poulain, J.-P. Mutations et modes alimentaires, in Paillat (dir.), Le mangeur et l'animal. Mutations de l'élevage et de la consommation. Éditions Autrement, coll. Mutations/mangeurs, n¹72, 1997, pp. 103-121.

(5) Le Marec, J., op. cit.

(6) Voir notamment Lévy-Leblond, J.-M. La science en mal de culture. Paris : Éditions Futuribles, Paris, 2004

(7) Le Marec, J. En guise d'épilogue - pratiques interprétatives : entre méthodes et sens commun, Études de communication, n²4, 2001, pp. 125-136. 